\title{
The joint universality and the generalized strong recurrence for Dirichlet $L$-functions
}

by

\author{
TAKASHI NAKAMURA (Tokyo)
}

1. Introduction and the statement of main results. The valuedistribution of the Riemann zeta function $\zeta(s)$ and Dirichlet $L$-functions $L(s, \chi)$ were investigated by numerous mathematicians (see for example [7]). In 1975, S. M. Voronin showed the universality theorem. To state it, we need some notation. Let meas $\{A\}$ denote the Lebesgue measure of the set $A$, and, for $T>0$, write $\nu_{T}\{\ldots\}:=T^{-1}$ meas $\{\tau \in[0, T]: \ldots\}$ where the dots stand for a condition satisfied by $\tau$. Let $D:=\{s \in \mathbb{C}: 1 / 2<\Re(s)<1\}$, and $K$ and $K_{1}, \ldots, K_{m}$ be compact subsets of the strip $D$ with connected complements.

The modern version of Voronin's theorem is as follows.

Theorem A (see [4, Theorem 6.5.2]). Let $f(s)$ be a non-vanishing continuous function on $K$ which is analytic in the interior of $K$. Then for every $\varepsilon>0$, we have

$$
\liminf _{T \rightarrow \infty} \nu_{T}\left\{\sup _{s \in K}|\zeta(s+i \tau)-f(s)|<\varepsilon\right\}>0 .
$$

Roughly speaking, this theorem implies that any non-vanishing analytic function can be uniformly approximated by the Riemann zeta function. As a generalization of Theorem A, Voronin also proved the joint universality theorem, which implies that a collection of Dirichlet $L$-functions with non-equivalent characters uniformly and simultaneously approximates nonvanishing analytic functions. In a slightly different form this was also established by Gonek and Bagchi, independently (both of these papers are unpublished doctoral theses). The strongest version of the joint universality for Dirichlet $L$-functions states:

Theorem B (see [7, Theorem 1.10]). Let $\chi_{1} \bmod q_{1}, \ldots, \chi_{m} \bmod q_{m}$ be pairwise non-equivalent Dirichlet characters, and $f_{l}(s)$ be a non-vanishing

2000 Mathematics Subject Classification: Primary 11M06; Secondary 11M26.

Key words and phrases: joint universality, Riemann zeta function, Dirichlet $L$-functions, generalized Riemann hypothesis, strong recurrence. 
continuous function on $K_{l}$ which is analytic in the interior of $K_{l}$ for $1 \leq$ $l \leq m$. Then for every $\varepsilon>0$, we have

$$
\liminf _{T \rightarrow \infty} \nu_{T}\left\{\sup _{1 \leq l \leq m} \sup _{s \in K_{l}}\left|L\left(s+i \tau, \chi_{l}\right)-f_{l}(s)\right|<\varepsilon\right\}>0 .
$$

Many mathematicians have considered generalizations of the universality (see for example [7]). On the other hand, B. Bagchi proved that the (generalized) Riemann hypothesis is true if and only if the Riemann zeta function (resp. Dirichlet $L$-functions) can be approximated by itself (resp. by Dirichlet $L$-functions) in the sense of universality.

Theorem C (see [1, Theorem 3.7]). The (generalized) Riemann hypothesis is true if and only if, for any $K$ and $\varepsilon>0$,

$$
\liminf _{T \rightarrow \infty} \nu_{T}\left\{\sup _{s \in K}|L(s+i \tau, \chi)-L(s, \chi)|<\varepsilon\right\}>0 .
$$

This property is called strong recurrence (see also [7, Theorem 8.3]). Another property similar to strong recurrence follows from Kaczorowski, Laurinčikas and Steuding [3] (see also [7, Section 10.6]).

Theorem D (see [3, Theorem 4]). Let $\mathcal{K}$ be a compact subset of $D$ with connected complement and let $\lambda \in \mathbb{R}$ be such that $\mathcal{K}$ and $\mathcal{K}+i \lambda:=\{s+i \lambda$ : $s \in \mathcal{K}\}$ are disjoint. Then

$$
\liminf _{T \rightarrow \infty} \nu_{T}\left\{\sup _{s \in \mathcal{K}}|L(s+i \lambda+i \tau, \chi)-L(s+i \tau, \chi)|<\varepsilon\right\}>0 .
$$

In this paper, we will show the following joint universality theorem.

TheOREM 1.1. Let $1=d_{1}, d_{2}, \ldots, d_{m}$ be algebraic real numbers linearly independent over $\mathbb{Q}$, and $d \in \mathbb{R} \backslash\{0\}$. Suppose $f_{l}(s)$ is a non-vanishing continuous function on $K_{l}$ which is analytic in the interior of $K_{l}$ for each $1 \leq l \leq m$. Then for every $\varepsilon>0$, we have

$$
\liminf _{T \rightarrow \infty} \nu_{T}\left\{\sup _{1 \leq l \leq m} \sup _{s \in K_{l}}\left|L\left(s+i d d_{l} \tau, \chi\right)-f_{l}(s)\right|<\varepsilon\right\}>0 .
$$

The assumption on $1=d_{1}, d_{2}, \ldots, d_{m}$ in Theorem 1.1 is essential (see the proof of Theorem 1.1 and Remark 2.6).

By putting $K:=K_{j}=K_{k}$ and $1 \equiv f_{j}(s) \equiv f_{k}(s)$ in Theorem 1.1, and using the triangle inequality $\left|L\left(s+i d d_{j} \tau, \chi\right)-L\left(s+i d d_{k} \tau, \chi\right)\right| \leq\left|L\left(s+i d d_{j} \tau, \chi\right)-1\right|+\left|L\left(s+i d d_{k} \tau, \chi\right)-1\right|$, we also obtain the following corollary, generalized strong recurrence.

COROllary 1.2. Let $d_{1}, \ldots, d_{m}$ and $d$ be as in Theorem 1.1. Then for any $K$ and $\varepsilon>0$, we have

$$
\liminf _{T \rightarrow \infty} \nu_{T}\left\{\sup _{s \in K}\left|L\left(s+i d d_{j} \tau, \chi\right)-L\left(s+i d d_{k} \tau, \chi\right)\right|<\varepsilon\right\}>0 .
$$


We will also show the next theorem and corollary, a kind of generalization of Theorem 1.1 and Corollary 1.2, respectively.

Theorem 1.3. Let $\delta_{1}=1, f_{1}(s), f_{2}(s)$ and $K_{1}, K_{2}$ be as in Theorem 1.1. Then for almost all $\delta_{2} \in \mathbb{R}$ and every $\varepsilon>0$, we have

$$
\liminf _{T \rightarrow \infty} \nu_{T}\left\{\sup _{1 \leq l \leq 2} \sup _{s \in K_{l}}\left|L\left(s+i \delta_{l} \tau, \chi\right)-f_{l}(s)\right|<\varepsilon\right\}>0 .
$$

Corollary 1.4. For almost all $\delta_{2} \in \mathbb{R}$ and for any $K$ and $\varepsilon>0$,

$$
\liminf _{T \rightarrow \infty} \nu_{T}\left\{\sup _{s \in K}\left|L(s+i \tau, \chi)-L\left(s+i \delta_{2} \tau, \chi\right)\right|<\varepsilon\right\}>0 .
$$

If we could take $\delta_{2}=0$ in (1.4), we could obtain strong recurrence, which is equivalent to the (generalized) Riemann hypothesis (see Theorem C).

2. Proof of main theorems. Firstly, we quote Baker's theorem.

Lemma 2.1 (see [2, Theorem 2.4]). The numbers $\alpha_{1}^{\beta_{1}} \cdots \alpha_{n}^{\beta_{n}}$ are transcendental for any algebraic numbers $\alpha_{1}, \ldots, \alpha_{n}$ other than 0 or 1 , and any algebraic numbers $\beta_{1}, \ldots, \beta_{n}$ with $1, \beta_{1}, \ldots, \beta_{n}$ linearly independent over the rationals.

Proposition 2.2. Let $p_{n}$ be the $n$th prime number and $1=d_{1}, d_{2}, \ldots, d_{m}$ be algebraic real numbers which are linearly independent over $\mathbb{Q}$. Then $\left\{\log p_{n}^{d_{l}}\right\}_{n \in \mathbb{N}}^{1 \leq l \leq m}$ is linearly independent over $\mathbb{Q}$.

Proof. Suppose the contrary,

$$
\sum_{n=1}^{r} c_{1 n} \log p_{n}+\sum_{n=1}^{r} c_{2 n} \log p_{n}^{d_{2}}+\cdots+\sum_{n=1}^{r} c_{m n} \log p_{n}^{d_{m}}=0, \quad c_{l n} \in \mathbb{Q} .
$$

By the above formula, we have

$$
p_{1}^{c_{11}} \cdots p_{r}^{c_{1 r}}=\left(p_{1}^{c_{21}} \cdots p_{r}^{c_{2 r}}\right)^{-d_{2}} \cdots\left(p_{1}^{c_{m 1}} \cdots p_{r}^{c_{m r}}\right)^{-d_{m}} .
$$

The left-hand side of (2.1) is an algebraic number. But the right-hand side of $(2.1)$ is transcendental when $\left(c_{21}, \ldots, c_{2 r}, \ldots, c_{m 1}, \ldots, c_{m r}\right) \neq(0, \ldots, 0)$ by Lemma 2.1 and the unique factorization of prime numbers. If $c_{k 1}=\cdots=$ $c_{k r}=0$, for some of $2 \leq k \leq m$, we can apply a lower-dimensional case of Baker's theorem. When $\left(c_{21}, \ldots, c_{2 r}, \ldots, c_{m 1}, \ldots, c_{m r}\right)=(0, \ldots, 0)$, we obtain $c_{11}=\cdots=c_{1 n}=0$ by the unique factorization of prime numbers.

In order to prove some limit theorem for the Riemann zeta function and Dirichlet $L$-functions, we will modify some definitions and theorems of [4, Section 5] and [7, Section 4]. Let $\mathfrak{B}(S)$ stand for the class of Borel sets of the space $S$. Denote by $H(D)$ the space of functions analytic on $D$, equipped with the topology of uniform convergence on compacta, and $H^{m}(D):=$ $H(D) \times \cdots \times H(D)$. Define on $\left(H^{m}(D), \mathfrak{B}\left(H^{m}(D)\right)\right)$ the probability measure $P_{T}(A):=\nu_{T}^{\tau}\left\{\left(L\left(s+i d_{1} \tau, \chi\right), \ldots, L\left(s+i d_{m} \tau, \chi\right)\right) \in A\right\}, \quad A \in \mathfrak{B}\left(H^{m}(D)\right)$. 
What we need is a limit theorem in the sense of weak convergence of probability measures for $P_{T}$ as $T \rightarrow \infty$, with an explicit form of the limit measure. Denote by $\gamma$ the unit circle on $\mathbb{C}$, and let $\Omega:=\prod_{p} \gamma(p)$, where $\gamma(p)=\gamma$ for all prime numbers. With the product topology and pointwise multiplication, the infinite-dimensional torus $\Omega$ is a compact topological Abelian group.

Denoting by $\underline{m}_{H}$ the probability Haar measure on $\left(\Omega^{m}, \mathfrak{B}\left(\Omega^{m}\right)\right)$, where $\Omega^{m}:=\Omega \times \cdots \times \Omega$, we obtain a probability space $\left(\Omega^{m}, \mathfrak{B}\left(\Omega^{m}\right), \underline{m}_{H}\right)$. Let $\omega_{l}(p)$ be the projection of $\omega_{l} \in \Omega$ to the coordinate space $\gamma(p)$, and define on the probability space $\left(\Omega^{m}, \mathfrak{B}\left(\Omega^{m}\right), \underline{m}_{H}\right)$ the $H^{m}(D)$-valued random element $\underline{L}(s, \chi, \underline{\omega}):=\left(L\left(s, \chi, \omega_{1}\right), \ldots, L\left(s, \chi, \omega_{m}\right)\right)$, where

$$
L\left(s, \chi, \omega_{l}\right):=\prod_{p}\left(1-\frac{\chi(p) \omega_{l}(p)}{p^{s}}\right)^{-1}, \quad s \in D, 1 \leq l \leq m .
$$

Let $P_{\underline{L}}$ stand for the distribution of the random element $\underline{L}(s, \chi, \underline{\omega})$, i.e.

$$
P_{\underline{L}}(A):=\underline{m}_{H}\left(\underline{\omega} \in \Omega^{m}: \underline{L}(s, \chi, \underline{\omega}) \in A\right), \quad A \in \mathfrak{B}\left(H^{m}(D)\right) .
$$

Proposition 2.3. The probability measure $P_{T}$ converges weakly to $P_{\underline{L}}$ as $T \rightarrow \infty$.

Proof. The key for the proof of [5, Theorem 1] and [6, Theorem 1] is the linear independence over $\mathbb{Q}$ of $\left\{\log \left(n+\alpha_{l}\right)\right\}_{n \in l \leq \mathbb{N}_{0}}^{1}$ resp. $\left\{\log p_{n}\right\}_{n \in \mathbb{N}} \cup$ $\{\log (n+\alpha)\}_{n \in \mathbb{N}_{0}}$, where $\mathbb{N}_{0}:=\mathbb{N} \cup\{0\}, \alpha \in \mathbb{R} \backslash \overline{\mathbb{Q}}$, and $\alpha_{1}, \ldots, \alpha_{m}$ are algebraically independent over $\mathbb{Q}$. In our case, $\left\{\log p_{n}^{d_{l}}\right\}_{n \in \mathbb{N}}^{1 \leq l \leq m}$ is linearly independent over $\mathbb{Q}$ by Proposition 2.2. Firstly, we have to show that the probability measure

$$
Q_{T}(A):=\nu_{T}\left\{\left(p_{n}^{i d_{1} \tau}, \ldots, p_{n}^{i d_{m} \tau}\right) \in A\right\}, \quad A \in \mathfrak{B}\left(\Omega^{m}\right),
$$

converges weakly to the Haar measure $\underline{m}_{H}$ on $\left(\Omega^{m}, \mathfrak{B}\left(\Omega^{m}\right)\right)$ as $T \rightarrow \infty$. We can show this by modifying the proof of [5, Lemma 4] or [6, Lemma 3] and using Proposition 2.2 (see also [4, Theorem 1.3.19] or [7, Lemma 4.4]). In addition, we can show that the one-parameter group $\left\{\Phi_{\tau}: \tau \in \mathbb{R}\right\}$, where

$$
\Phi_{\tau}(\underline{\omega}):=\left(p_{n}^{i d_{1} \tau} \omega_{1}\left(p_{n}\right), \ldots, p_{n}^{i d_{m} \tau} \omega_{m}\left(p_{n}\right)\right),
$$

is ergodic, by modifying the proof of [5, Lemma 9] or [6, Lemma 7] and using Proposition 2.2 (see also [4, Theorem 5.3.6] or [7, Lemma 4.7]). Hence we obtain the desired assertion by modifying the proof of $[5$, Theorem 1$]$ or $[6$, Theorem 1].

The next lemmas have been obtained only in the case $m=1$ (see for example $[4$, Section 6] or [7, Section 5]). 
Lemma 2.4. Let $\left\{\underline{x}_{n}\right\}$ be a sequence in $\mathbb{C}^{m}$ satisfying $\sum_{n=1}^{\infty}\left|\underline{x}_{n}\right|^{2}<\infty$. Then there exists a sequence $\left\{\underline{b}_{n}^{\prime}\right\}$ with $\underline{b}_{n}^{\prime} \in\{ \pm 1, \ldots, \pm 1\}$ such that the series $\sum_{n=1}^{\infty}\left(b_{1 n} x_{1 n}, \ldots, b_{m n} x_{m n}\right)$ converges.

Proof. The case $m=1$ coincides with [4, Lemma 6.5.3] or [7, Lemma 5.5]. Now use this case for each component of $\sum_{n=1}^{\infty}\left(b_{1 n} x_{1 n}, \ldots, b_{m n} x_{m n}\right)$.

Lemma 2.5. Let $\left\{\underline{f}_{n}\right\}$ be a sequence in $H^{m}(D)$ which satisfies:

(a) If $\mu_{l}$ are complex measures on $(\mathbb{C}, \mathfrak{B}(\mathbb{C}))$ with compact support contained in $D$ such that $\sum_{n=1}^{\infty}\left|\int_{\mathbb{C}} f_{l n} d \mu_{l}\right|<\infty$, then $\int_{\mathbb{C}} s^{r} d \mu_{l}(s)=0$ for all $1 \leq l \leq m$ and $r \in \mathbb{N}_{0}$.

(b) The series $\sum_{n=1}^{\infty} \underline{f}_{n}$ converges in $H^{m}(D)$.

(c) For any compact set $\mathcal{K}_{l} \subset D, \sum_{n=1}^{\infty} \sup _{1 \leq l \leq m} \sup _{s \in \mathcal{K}_{l}}\left|f_{l n}(s)\right|^{2}<\infty$. Then the set of all convergent series $\sum_{n=1}^{\infty}\left(a_{1 n} f_{1 n}, \ldots, a_{m n} f_{m n}\right)$ with $\left|a_{l n}\right|$ $=1$ is dense in $H^{m}(D)$.

Proof. For $m=1$, the assertion has been proved in [4, Theorem 6.3.10] or [7, Theorem 5.7]. Now apply this case to each component of

$$
\sum_{n=1}^{\infty}\left(a_{1 n} f_{1 n}, \ldots, a_{m n} f_{m n}\right) .
$$

Proof of Theorem 1.1. In view of the standard proof of universality (see $[4$, Section 6$]$ or $[7$, Section 5]), we only have to check the three assumptions of Lemma 2.5. Assumption (a) holds by [4, Theorem 6.4.14] (see the proof of [4, Lemma 6.5.4] or [7, Theorem 5.10]). Lemma 2.4 yields (b), and (c) is obvious because $1 / 2<\Re(s)<1$.

Therefore we obtain the assertion for $d=1$ by modifying the proof of [4, Theorem 6.5.2] or [7, Theorem 5.14], which are one-dimensional universality theorems. For the multi-dimensional case, we refer to the proof of $[6$, Theorem 2], which is the two-dimensional universality theorem.

Finally, by changing the parameter $\tau \mapsto d \tau$, we obtain Theorem 1.1.

REMARK 2.6. We have examples for which (1.1) is not true when $d_{1}, d_{2}$ are linearly dependent over $\mathbb{Q}$. For instance, the case $d_{2}=-1$ is proved as follows. Let $K_{1}=K_{2}$ be a one-point set on the real axis in $D$. In this case, any $\tau$ satisfying $|L(\sigma+i \tau, \chi)+i|<\varepsilon$ must satisfy $|L(\sigma-i \tau, \chi)-i|<\varepsilon$ for any real Dirichlet character.

It should be noted that $1, d d_{1}, d d_{2}$ are not always linearly independent over $\mathbb{Q}$ when $1, d_{1}, d_{2}$ are linearly independent over $\mathbb{Q}$. For instance $1, d \sqrt{2}$ and $d \sqrt{3}$ are linearly dependent over $\mathbb{Q}$ when $d^{-1}=\sqrt{2}+\sqrt{3}$.

Next, we will show Theorem 1.3.

Lemma 2.7. For almost all $\delta_{2} \in \mathbb{R},\left\{\log p_{n}\right\} \cup\left\{\log p_{n}^{\delta_{2}}\right\}$ is linearly independent over $\mathbb{Q}$. 
Proof. Suppose the contrary. In view of proof of Proposition 2.2, we have

$$
p_{1}^{c_{11}} \cdots p_{r}^{c_{1 r}}=\left(p_{1}^{c_{21}} \cdots p_{r}^{c_{2 r}}\right)^{-\delta_{2}} .
$$

The left-hand side of (2.3) is an algebraic number. But the right-hand side of $(2.3)$ is transcendental when $\left(c_{21}, \ldots, c_{2 r}\right) \neq(0, \ldots, 0)$ for almost all $\delta_{2} \in$ $\mathbb{R}$ by the unique factorization of prime numbers and the fact that almost all real numbers are transcendental. Hence the right-hand side of (2.3) is transcendental except for a null set of $\delta_{2} \in \mathbb{R}$. The set of algebraic numbers is countable, and a countable union of null sets is also a null set. Thus for all $0<q \in \overline{\mathbb{Q}} \backslash\{1\}$ and for almost all $\delta_{2} \in \mathbb{R}, q^{\delta_{2}}$ is transcendental. Therefore we obtain the conclusion by modifying the proof of Proposition 2.2.

Proof of Theorem 1.3 and Corollary 1.4. By using Lemma 2.7 instead of Proposition 2.2, and modifying the proof of Theorem 1.1, we obtain Theorem 1.3. We also obtain Corollary 1.4 by using the method of proof of Corollary 1.2 .

REMARK 2.8. Theorems 1.1 and 1.3 can be generalized to the Dirichlet series belonging to the class $\tilde{\mathcal{S}}$ introduced in [7] (see [7, Section 2.2 and Notation]). Roughly speaking, $\tilde{\mathcal{S}}$ is the class of Dirichlet series satisfying the Ramanujan hypothesis and a condition similar to the prime number theorem, continued analytically, of finite order, and having polynomial Euler product.

Acknowledgments. I thank Professor Kohji Matsumoto and the referee for very useful advice.

\section{References}

[1] B. Bagchi, A joint universality theorem for Dirichlet L-functions, Math. Z. 181 (1982), 319-334.

[2] A. Baker, Transcendental Number Theory, Cambridge Math. Lib., Cambridge Univ. Press, Cambridge, 1975.

[3] J. Kaczorowski, A. Laurinčikas and J. Steuding, On the value distribution of shifts of universal Dirichlet series, Monatsh. Math. 147 (2006), 309-317.

[4] A. Laurinčikas, Limit Theorems for the Riemann Zeta-Function, Math. Appl. 352, Kluwer, Dordrecht, 1996.

[5] A. Laurinčikas and K. Matsumoto, Joint value-distribution theorems on Lerch zetafunctions II, Lithuanian Math. J. 46 (2006), 271-286.

[6] H. Mishou, The joint value-distribution of the Riemann zeta function and Hurwitz zeta functions, ibid. 47 (2007), 32-47.

[7] J. Steuding, Value-Distribution of L-functions, Lecture Notes in Math. 1877, Springer, Berlin, 2007.

Department of Mathematics, Faculty of Science and Technology

Tokyo University of Science, Noda, Chiba 278-8510, Japan

E-mail: nakamura_takashi@ma.noda.tus.ac.jp 